\title{
Spatial population genetic structure and colony dynamics in Damaraland mole-rats (Fukomys damarensis) from the southern Kalahari
}

\author{
Samantha Mynhardt ${ }^{1,2^{*}}$, Lorraine Harris-Barnes ${ }^{1}$, Paulette Bloomer ${ }^{1,3}$ and Nigel C. Bennett ${ }^{2,3}$
}

\begin{abstract}
Background: Non-random associations within and among groups of social animals can provide valuable insight into the function of group living and the evolution of social behaviour. Damaraland mole-rats (Fukomys damarensis) demonstrate extremely high levels of reproductive skew, and dispersal is considered to be male-biased in onset and frequency, although asymmetry in dispersal distance is yet to be investigated. Dispersal may be positively correlated with increasing favourable environmental conditions, such as rainfall, however, the effects of ecological constraints on dispersal and colony fission-fusion dynamics have not previously been demonstrated on a spatial scale. Here we provide the first spatial population genetic study for this species. We investigated genetic structure in a population of Damaraland mole-rats from the southern Kalahari in South Africa over 3 years, combining observational dispersal data from mark-recapture with population genetic data to evaluate (1) sex-bias in frequency and distance of dispersal in this species, and (2) the effect of rainfall on fission-fusion dynamics of colonies.

Results: Our results demonstrate (1) that both males and females favour local dispersal but on rare occasions may disperse over distances greater than $400 \mathrm{~m}$, (2) that males may disperse over greater distances than females, and (3) that males more frequently immigrate into established neighbouring colonies than females, who predominantly disperse by colony fission, i.e. multiple individuals "budding" from their native colony into a neighbouring territory, thereby establishing new colonies. Furthermore, our results demonstrate (4) elevated dispersal and colony fission in association with increased rainfall, supporting the hypothesis that rainfall may play a significant role in the maintenance and/or disruption of reproductive skew in Damaraland mole-rat populations.
\end{abstract}

Conclusion: This study represents the first fine-scale spatial population genetic study in Damaraland mole-rats, and provides relevant insights into colony fission-fusion dynamics in a social and cooperatively breeding species.

Keywords: Social mammal, Sex-biased dispersal, Dispersal distance, Ecological constraints, Reproductive skew, Population genetics

*Correspondence: samantha.mynhardt@up.ac.za

${ }^{1}$ Molecular Ecology and Evolution Programme, Department

of Biochemistry, Genetics and Microbiology, University of Pretoria,

Pretoria, South Africa

Full list of author information is available at the end of the article

\section{Background}

Investigating non-random associations within and among groups of social animals is essential for understanding the function of group living and the evolution of social behaviour. Population subdivision in social species may result in the formation of smaller groups, or "colonies". These colonies are not necessarily defined by their 
geographical site, but rather by their social interactions, including affinities, antagonisms, hierarchy and kinship [1]. Philopatry in these species would refer to fidelity to natal colony rather than to natal environment, and similarly dispersal refers to movement and/or integration of individuals from one social group into another. Colonies may grow or shrink over time and space, split (fission) or merge (fusion) with other colonies.

Philopatry and dispersal have been extensively studied in social species and frequently address the question of asymmetry in dispersal patterns among sexes. Dispersal asymmetry depends on social organization; in mammals, male-biased dispersal and female philopatry is common, while reversed asymmetry involving female-biased dispersal is rare, and more typically observed in passerine birds [2,3]. Numerous population genetics studies have addressed the consequences of female philopatry, male dispersal and matrilineal structure for genetic differentiation between groups. These include theoretical $[4,5]$ and empirical studies involving primates [6, 7]; bats [8,9]; plateau pikas [10] and woodrats [11, 12]. Demographic events (births, deaths, migrations) drive the size and composition of colonies, and optimal colony sizes vary among species, populations and environments [13-15]. Colony fission may occur when colonies reach a maximum size, thus producing two or more new colonies, as reported in diverse species including social spiders [16], army ants [17], naked mole-rats [18, 19], marmots [20, $21]$ and primates $[22,23]$.

The African mole-rat family (Bathyergidae) displays a wide range of sociality and co-operative breeding strategies among species (reviewed in [24]). Damaraland molerats (Fukomys damarensis) demonstrate extremely high levels of skew in reproductive success, with less than $8 \%$ of individuals typically achieving direct reproductive success [25]. Populations of Damaraland mole-rats are traditionally thought to be structured into extended family groups, or colonies of variable sizes ranging from 2 to 40 individuals, typically comprising a single breeding female (the queen), together with one or two breeding males, and their offspring $[24,26]$. Non-breeding colony members form part of the subordinate work force and contribute to the care of the young [27]. These subordinates may be reproductively suppressed, but are not sterile [28]. Breeding pairs are in most instances unrelated and are usually the founders of the colonies in which they breed [26].

The evolution of cooperative breeding in the Bathyergidae may be linked to habitat aridity and food distribution [27, 29-33]. The Damaraland mole-rat is endemic to the arid ecoregions of southern Africa [26]. The habitat is characterized by high temperatures, low and sporadic rainfall patterns and coarse sandy soils, typically including the thorn-scrub woodland savannas and grasslands [27, 34]. These arid habitats with low rainfall and hard, coarse soils elevate the energetic cost of burrowing, and coupled with the uneven distribution of food resources in the form of underground roots and swollen tubers of geophytes, may result in reduced foraging success [27, 31]. This could lead to selection for group living, cooperative foraging and communal care of offspring, resulting in the evolution of the extreme reproductive skew seen in this species.

Damaraland mole-rats may disperse between colonies, either temporarily or permanently, either above or below ground [35]. The success of dispersal in these harsh habitats, is dependent on predation risk [25, 29], availability of resources and the distances between neighbouring colonies [35, 36], and has been found to be male-biased $[36,37]$. Despite the risks associated with dispersal, these animals seem to disperse regularly enough to maintain reasonable levels of outbreeding in most wild populations, probably since dispersal is one of the only means by which non-breeding individuals can gain reproductive opportunity with prospective unrelated mates [38].

Various ecological and demographic factors have been proposed as drivers of dispersal in mole-rats. First, escape of the dominant breeders' control in an individual's native colony, in order to exploit his/her lifetime reproductive success and mate with non-colony affiliates [39]. Secondly, an attempt at inbreeding avoidance by circumventing reproduction with related colony affiliates [38], and thirdly, favourable environmental conditions such as resource availability, rainfall and good soil quality, promoting dispersal [27]. Previous studies have suggested that the social structure and reproductive patterns in Damaraland mole-rats are heavily influenced by the environment they occur in $[26,27,29,35,37,40]$. The frequency of dispersal is thought to be positively correlated with increasing favourable environmental conditions, such as rainfall $[25,27,35,37,41]$, but field data showing how ecological constraints affect dispersal are scarce [24, 37, 42], and the effect of ecological constraints on dispersal and colony fission-fusion dynamics has not been demonstrated on a spatial scale. Torrents-Ticó et al. [37] demonstrate that rainfall increases dispersal probability in both sexes, with males dispersing earlier and more frequently than females, however dispersal distances were not investigated. Thus, asymmetry among sexes in dispersal distance, as well as the nature of spatial population genetic structure in this species remain largely unknown.

Here we investigate spatial population genetic structure in Damaraland mole-rats, and combine genetic data with direct observational dispersal data through mark-recapture to evaluate (1) asymmetry in frequency 
and distance of dispersal in this species, and (2) the effect of rainfall on fission-fusion dynamics of colonies. We distinguish colony fission as multiple individuals "budding" from their native colony into a neighbouring territory, from regular dispersal, typically involving a single individual dispersing into another established colony or joining another individual to form a new colony. We studied a population of Damaraland mole-rats, comprising 74 colonies, in a $1700 \mathrm{~m} \times 500 \mathrm{~m}$ study site in the Tswalu Nature Reserve in the southern Kalahari of South Africa, over a period of 3 years (2004-2006). Genetic methods offer a means of quantifying dispersal that avoids the spatial biases associated with observational data, and may more accurately reflect the long-term average pattern of sex-biased dispersal [e.g. 43-45]. However, differences in estimates from genetic vs. observational data may arise in part due to varying reproductive success of dispersers, since genetic methods effectively measure gene flow, i.e. dispersal of gametes, or genes. A combination of direct observational data and indirect population genetic data may thus provide the most robust estimates of sex-biased dispersal [e.g. 46, 47]. In cooperatively breeding species, characterizing the nature of sex differences in dispersal is particularly important for understanding localized patterns of kin structure and sex-specific patterns of cooperation and conflict [48-50], and more broadly, these characterizations can assist in evaluating competing hypotheses for the evolution of sex-biased dispersal.

\section{Results}

\section{Dispersal}

Mark-recapture data revealed slight geographical shifts in location and/or range of various colonies that persisted from 1 sampling year to the next (e.g. see "Colossus" in Fig. 1). Mark-recapture data also revealed movements of individuals between colonies, primarily from 1 year to the next, but in some cases within a single year. For example, sample \#113 was captured at both colonies Tuareg (TUA) and Hodges (HOD) in 2005 (Fig. 1, Additional file 1: Table S1). A total of 16 male dispersers, representing 19 dispersal events (two individuals, \#64 and \#400, dispersed multiple times), and 17 female dispersers were recorded. Remarkably, sample \#64 was captured at Colossus (COL) and Zappa (ZAP) in 2004, Colossus and X-Men (XME) in 2005 and Andersens (AND) in 2006, revealing an unusually high dispersal frequency (three dispersals in 3 years) in

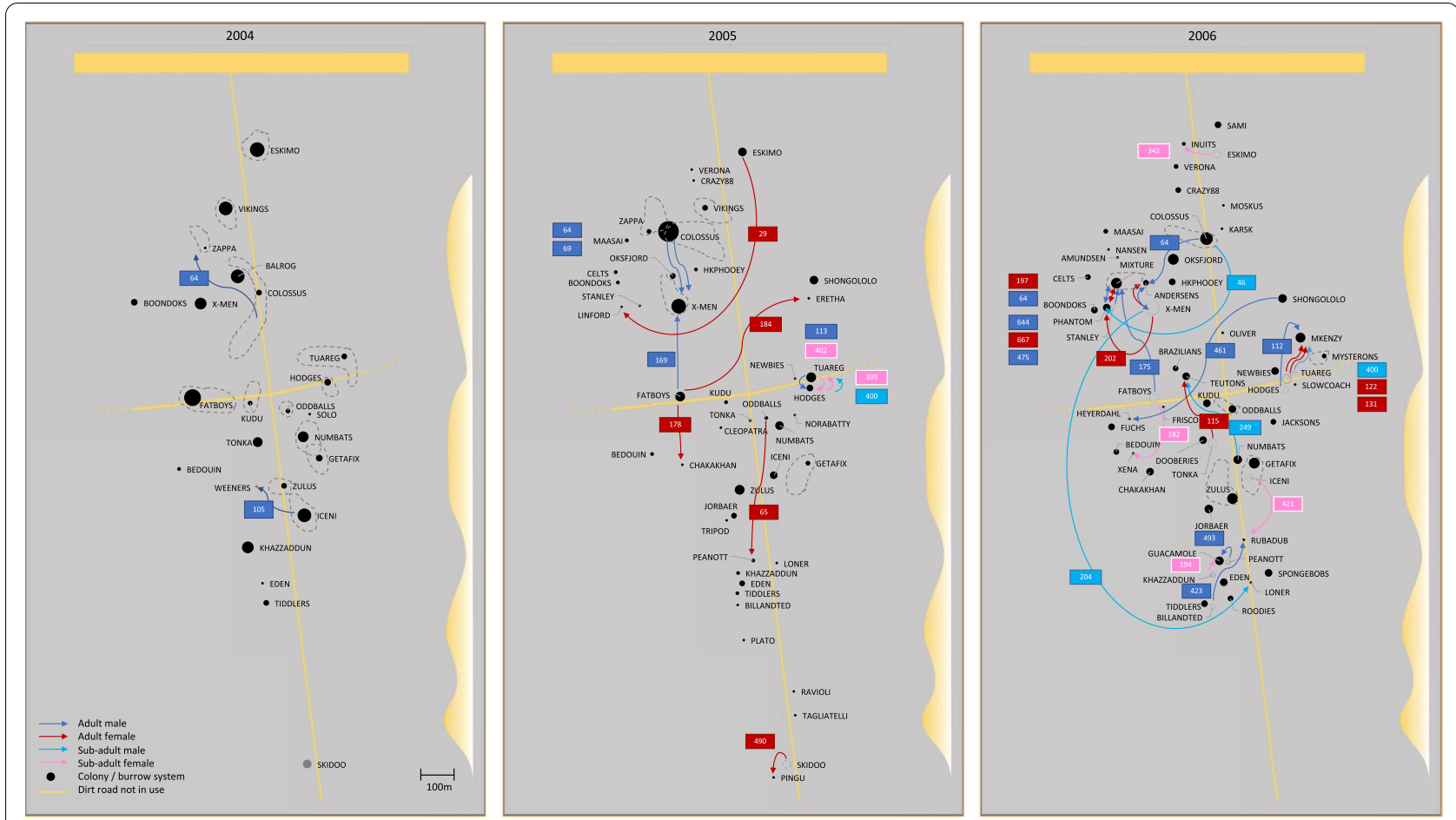

Fig. 1 Schematic representation of the geographical distribution of colonies within the $500 \mathrm{~m} \times 1700 \mathrm{~m}$ study site in Tswalu Kalahari Reserve. Colonies are depicted as black circles, with sizes representing sample size (ranging from 1 to 20 individuals in a given year). Colonies representing multiple sampling holes are depicted by grey dashed lines, and grey dotted circles indicate colonies not sampled (i.e. not present) in a given year. Dispersal events are indicated by coloured arrows representing adult males (dark blue), adult females (red), sub-adult males (light blue) and sub-adult females (pink), with coloured number-tags depicting the individual ID of each disperser 
this particular individual. Some neighbouring colonies showed a higher frequency of dispersals than others (e.g. see Tuareg and Hodges and Mkenzy, and Mixture, Phantom and Stanley; Fig. 1). All dispersal events are illustrated in Fig. 1, in which the first panel depicts movements during the 2004 sampling period, the second depicts movements since 2004 and within the 2005 sampling period, and the third depicts movements since 2005 and within the 2006 sampling period.

On average, males dispersed farther than females (mean \pm SE males: $215 \mathrm{~m} \pm 180 \mathrm{~m}$; females: $139 \mathrm{~m} \pm 104 \mathrm{~m}$; Fig. 2a) although this difference is nonsignificant $\left(t_{(16)}=1.7 ; p=0.11\right)$. Of the 36 (19 male; 17 female) dispersal events, only three represented distances greater than $400 \mathrm{~m}$ (\#64: $456 \mathrm{~m}$; \#461: $472 \mathrm{~m}$; \#204: $530 \mathrm{~m}$; Fig. 1, Additional file 1: Table S1), and all of these were males. Female dispersals were typically associated with establishment of new colonies (Fig. 1, Additional file 1: Table S1), with only two cases of dispersal into neighbouring colonies (12\% of female dispersal events), both of which were represented by very short distances (\#399 and \#402; Fig. 1, Additional file 1: Table S1). Males were found to disperse more frequently into established colonies, with six such cases recorded (32\% of male dispersal events).

\section{Population-level $F_{\mathrm{ST}}$ analysis}

Across all 3 sampling years, mean $F_{\mathrm{ST}}$ for the 47 colonies, calculated using 399 individuals, was high (0.167; Table 2), indicating substantial genetic differentiation among colonies, which may be expected in cooperatively breeding species, where individuals within any given colony are on average more related to one another than they are to other individuals in the population. Females showed a higher mean $F_{\mathrm{ST}}$ than males (females: 0.175; males: 0.153; Table 2), indicating significantly greater genetic differentiation among females than males $(p<0.001$; Table 2$)$, a pattern consistent with females being the more philopatric sex, and males being more dispersive.

When the three datasets (2004, 2005 and 2006) were analysed separately, $F_{\mathrm{ST}}$ was found to increase with increasing

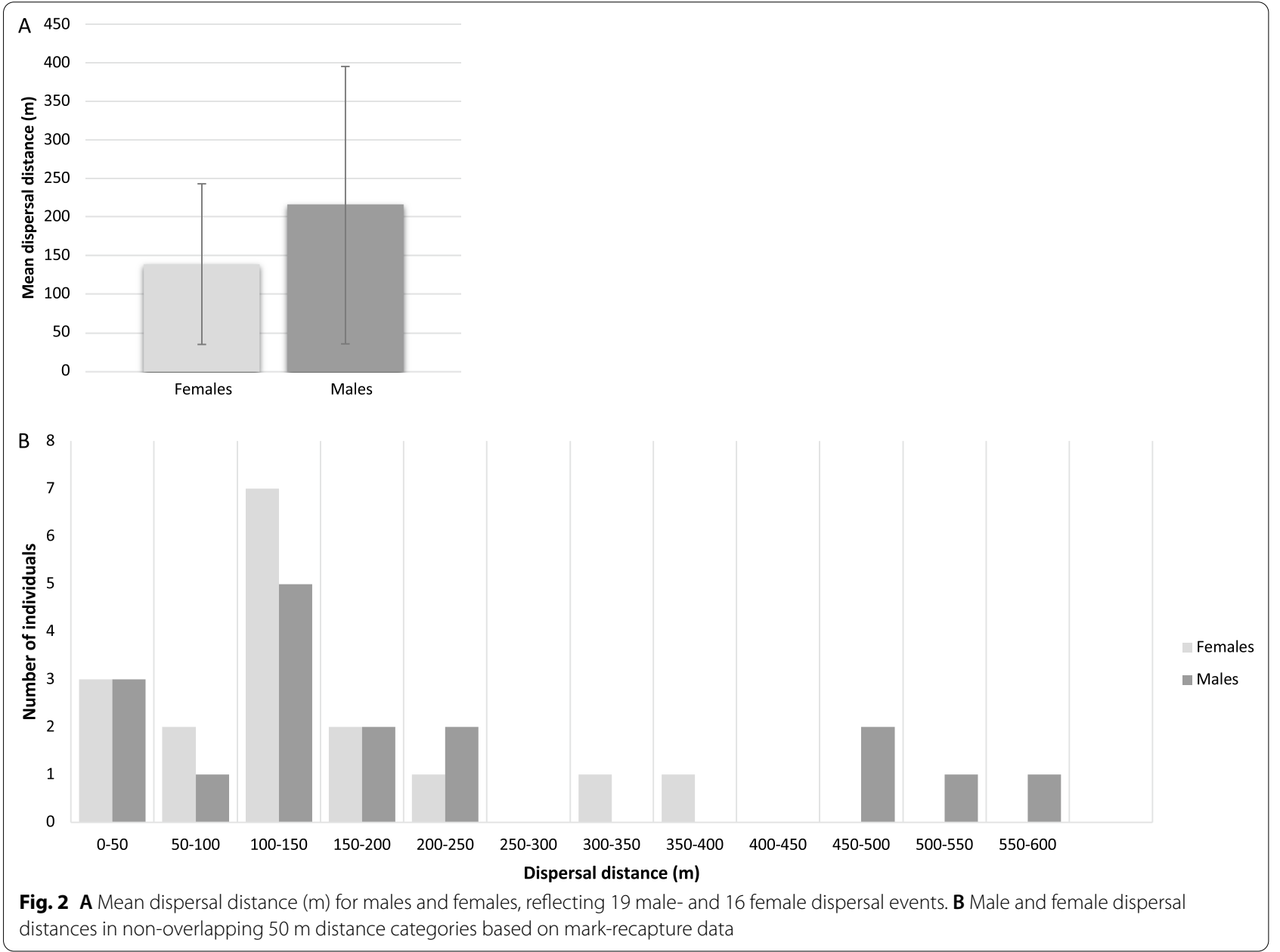


spatial distance in both sexes in 2004, however, over short distances $F_{\mathrm{ST}}$ among males was higher than females, and over increasing distances $F_{\mathrm{ST}}$ became higher among females than males (Fig. 3A). This trend resulted in a nonsignificant overall difference in $F_{\mathrm{ST}}$ between males and females $\left(p=0.108\right.$; Table 2). In 2005, $F_{\mathrm{ST}}$ increased with increasing spatial distance in males, but remained relatively constant (on average) in females (Fig. 3B). $F_{\mathrm{ST}}$ was higher among females than males across all distance classes (except in the 700-800 m distance class). Overall, in 2005, $F_{\mathrm{ST}}$ was significantly higher among females than males $\left(p<0.001\right.$; Table 2). In 2006, $F_{\mathrm{ST}}$ increased with increasing spatial distance in females, but decreased in males (Fig. 3C). $F_{\mathrm{ST}}$ was higher among females than males across all distance classes (except in the 100-200 m distance class, where they did not differ significantly). Overall, in 2006, $F_{\mathrm{ST}}$ was significantly higher among females than males $(p<0.001$; Table 2$)$.

\section{Spatial autocorrelation of individual relatedness}

Spatial autocorrelation analysis revealed significant positive genetic structure within colonies, among both males and females (Fig. 4; see $0 \mathrm{~m}$ distance in all three datasets, A-C, and overall, D), indicating a higher level of relatedness within colonies than would be expected under random mating, which is expected in cooperatively breeding species. Across all 3 sampling years, the mean intra-colony relationship coefficient, $r$, for the 47 colonies, calculated using 399 individuals, was high (0.290; Table 2). In contrast, inter-colony $r$ was negative $(-0.022$; Table 2$)$, indicating a low level of relatedness among colonies, also in line with expectations for this species.

Intra-colony $r$ was significantly higher among females than males in 2004 (females: 0.322; males: 0.287; $p=0.023$; Table 2) and in 2005 (females: 0.327; males: 0.189; $p=0.002$; Table 2), and did not differ significantly between sexes in 2006. On average, across all 3 years, intra-colony $r$ was significantly higher among females than males (females: 0.315; males: 0.264; $p=0.022$; Table 2). In all 3 years, male-female intra-colony $r$ values were between those of males and females (2004: $r=0.305 ; 2005: r=0.248$; 2006: $r=0.292$ ). Inter-colony $r$ was slightly higher among males than females in each year, and significantly higher on average across all 3 years (males: -0.020 ; females: -0.026 ; $p=0.011$; Table 2).

\section{Isolation by distance}

In all 3 years, intra-colony $r$ was high (mean 0.2899 ) and inter-colony $r$ low (mean -0.022), irrespective of distance between colonies (Table 2, Fig. 4). Linear regression, i.e. slope of the relationship between spatial distance and genetic relatedness, across all distance classes (excluding intra-colony) was low (not significantly different from zero; Table 2) for all datasets, hence we did not find evidence for IBD. However, it is worth noting that negative regression (blog) was consistently more pronounced among females than males (Table 2; Fig. 4).

\section{Dispersal estimates from genetic data}

Effective population density, $D$, was estimated for each dataset based on the number of individuals captured in the $0.85 \mathrm{~km}^{2}(1700 \mathrm{~m} \times 500 \mathrm{~m})$ study site. Neighbourhood size $(\mathrm{Nb})$ and sigma $(\sigma)$ estimates were obtained for the 2004 and 2006 male datasets, and the dataset comprising all males from all 3 years, while estimates for the female datasets and the 2005 male dataset did not converge, and therefore could not be estimated (Table 2). Sigma $(\sigma)$ estimates of $0.135,0.207$ and 0.135 for the 2004, 2006 and combined male datasets respectively translated to dispersal distance estimates of $190 \mathrm{~m}, 293 \mathrm{~m}$ and $191 \mathrm{~m}$ respectively.

\section{Effects of ecological constraints on dispersal and colony fission}

Average annual rainfall in Tswalu for the 6 years preceding the current study period (1998-2003) was $300.5 \mathrm{~mm} \pm 110.8 \mathrm{~mm}$. Annual rainfall for 2004, 2005 and 2006 was $247.9 \mathrm{~mm}, 422.7 \mathrm{~mm}$ and $532.6 \mathrm{~mm}$ respectively. Thus, rainfall in 2004 was below average, 2005 above average, and 2006 substantially above average.

Our mark-recapture data revealed 36 dispersal events across the 3 sampling years, of which two were detected within the 2004 sampling period, 11 between 2004 and 2005, 21 between 2005 and 2006, and a further two dispersals some time between 2004 and 2006 (these individuals were not captured in 2005, and therefore it is impossible to say exactly when they dispersed; Fig. 1; Additional file 1: Table S1). Since we do not have dispersal data preceding 2004, we could only compare observed dispersal from 2004 to 2005 with that of 2005 to 2006, and this test revealed a significant increase in the number of dispersal events between these sampling periods $(P=0.016)$.

(See figure on next page.)

Fig. 3 Population-level $F_{\text {ST }}$ for the 47 colonies analysed in this study, including 23 colonies in 2004 (A), 27 colonies in 2005 (B), and 34 colonies in 2006 (C). A total of 13 distance classes of $100 \mathrm{~m}$ increments were specified since the maximum distance between any two colonies in our study site was $1227 \mathrm{~m}$. Points represent mean $F_{\text {ST }}$ for each distance class, for males and females, and error bars represent $95 \%$ confidence intervals. Dotted lines represent linear trendlines for males (black) and females (grey) 

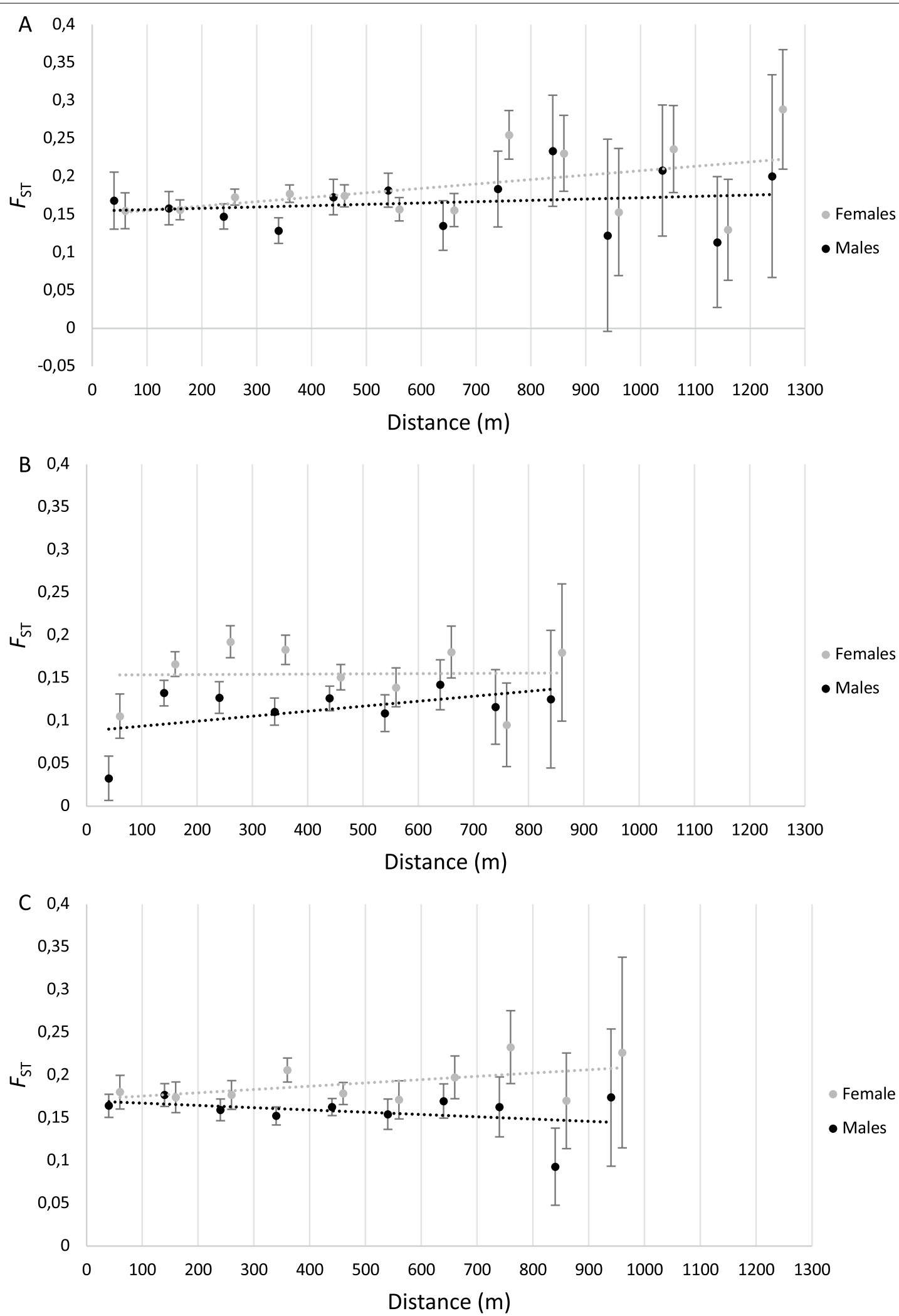

Fig. 3 (See legend on previous page.)

Females

- Males

- Female

- Males 
In our study population, 2004 was associated with relatively few colonies of large sizes, while 2005 and 2006 were characterized by successively larger numbers of smaller colonies, comprising many newly established colonies (Fig. 5). In 2004, a relatively small number of colonies [26] of relatively large size (mean = eight) existed, most of which persisted in $2005(19 / 26=73 \%)$, along with the addition of a few [21] newly established colonies (total $=40$ ), some arising from colony fission (Figs. 5 and 6). Average colony size in 2005 dropped from eight to four. In 2006, we observed further colony fission, with large established colonies decreasing in size as a result of emigration of colony members, colonies founded in 2005 increasing in size due to reproduction (in total 20 established colonies persisted, of which 12 had persisted since 2004), and yet more new colonies being established and already at a fair size due to reproduction. The average colony size thus rose again to 4.5 in 2006, although with many [28] newly established colonies (total $=48$ ), resulting in a substantial increase in overall population size. Thus, we also observed a significant increase in the number of newly established colonies, i.e. colony fission events $(P=0.001)$.

\section{Discussion}

\section{Spatial genetic structure and local dispersal}

Both population-level $F_{\mathrm{ST}}$ analysis and individual-level spatial autocorrelation analysis revealed strong signals of population genetic structure, with high levels of relatedness within colonies. This is not unexpected in a cooperatively breeding species, and is commonly associated with natal philopatry in social vertebrates, in which offspring of one or both sexes frequently inherit the breeding position in their natal group [51-56].

We did not find evidence for IBD at the spatial scale investigated here. Spatial autocorrelation of relatedness revealed that relatedness among individuals from colonies in close proximity, less than $200 \mathrm{~m}$ apart is not substantially higher than that of individuals from colonies at intermediate distances up to $800 \mathrm{~m}$ apart, but is instead relatively constant (close to zero) across this spatial range, and only begins to decrease at distances greater than $800 \mathrm{~m}$ (Fig. 4). Thus, it seems plausible that gene flow is well maintained at distances of up to $800 \mathrm{~m}$, and that IBD may become significant at greater distances, however this hypothesis is yet to be tested on a dataset representing a larger geographic range.

Observational data revealed a mean dispersal distance of $177 \mathrm{~m} \pm 150 \mathrm{~m}$, which is well below the mean distance between colonies in our site $(363 \mathrm{~m} \pm 210 \mathrm{~m})$, indicating that both males and females typically disperse to nearby colonies, rather than undertaking long distance dispersal. However, since this finding is not explicitly supported by the genetic data, the frequency of long-distance dispersals may be underestimated, particularly given the possibility that some individuals may disperse out of the study area. In our study few individuals undertook long-distance dispersals greater than $400 \mathrm{~m}$ (sample \#64: $456 \mathrm{~m}$; \#461: 472 m; \#204: 530 m; Additional file 1: Table S1, Fig. 1). Since these and other dispersals cross the territories of multiple other established colonies, it is likely that these may represent aboveground dispersal events. Finn [57] hypothesized that dispersal distances greater than $250 \mathrm{~m}$ are likely to represent aboveground dispersal in Damaraland mole-rats, due to the high energetic costs associated with digging. Long-distance dispersal can thus be risky, exposing individuals to predation, aggressive interactions with conspecifics, loss of body condition and stress $[31,39,58]$, while also trading off against cooperative contributions that they might otherwise have made within their natal group [59]. Local dispersal may also involve fitness costs, arising from kin competition [60] and/or exposure to a risk of inbreeding $[61,62]$. On the other hand, local dispersal could be facilitated by familiarity with individuals in the destination group [63], while colony fission, leading to the establishment of new breeding colonies in close proximity to the natal colony could be facilitated if relatives within the natal group were more accepting than potentially aggressive and territorial non-relatives elsewhere $[61,64]$. The high frequency of dispersals between colonies Hodges, Tuareg and Mkenzy could be a reflection of this type of local dispersal between neighbouring colonies, with the establishment of the new colony, Mkenzy, in 2006 (Fig. 1). More specifically, the movement of a male and female (\#113 and \#204; Fig. 1, Additional file 1: Table S1) from Tuareg to Hodges, and the converse (\#399 and \#400; Fig. 1, Additional file 1: Table S1), could indicate that individuals (possibly potential breeding pairs) are dispersing together. Torrents-ticó

(See figure on next page.)

Fig. 4 Spatial autocorrelation for the 399 individuals from 47 colonies analysed in this study, including 193 individuals from 23 colonies in 2004 (A), 146 individuals from 27 colonies in 2005 (B), 208 individuals form 34 colonies in 2006 (C), and all three datasets (2004, 2005, 2006) combined (D). A total of 13 distance classes of $100 \mathrm{~m}$ increments were specified, since the maximum distance between any two colonies in our study site was $1227 \mathrm{~m}$. Intra-colony estimates are indicated at $0 \mathrm{~m}$ distance. Points represent mean spatial autocorrelation coefficients for each distance class, for males and females, and error bars represent 95\% confidence intervals. Error bars that do not overlap zero represent significant genetic structure. Dotted lines represent linear trendlines for males (black) and females (grey). D The slope of regression (change in $r$ per $\mathrm{km}$ ) is steeper overall for females $(-0.1)$ than for males $(-0.08)$ 

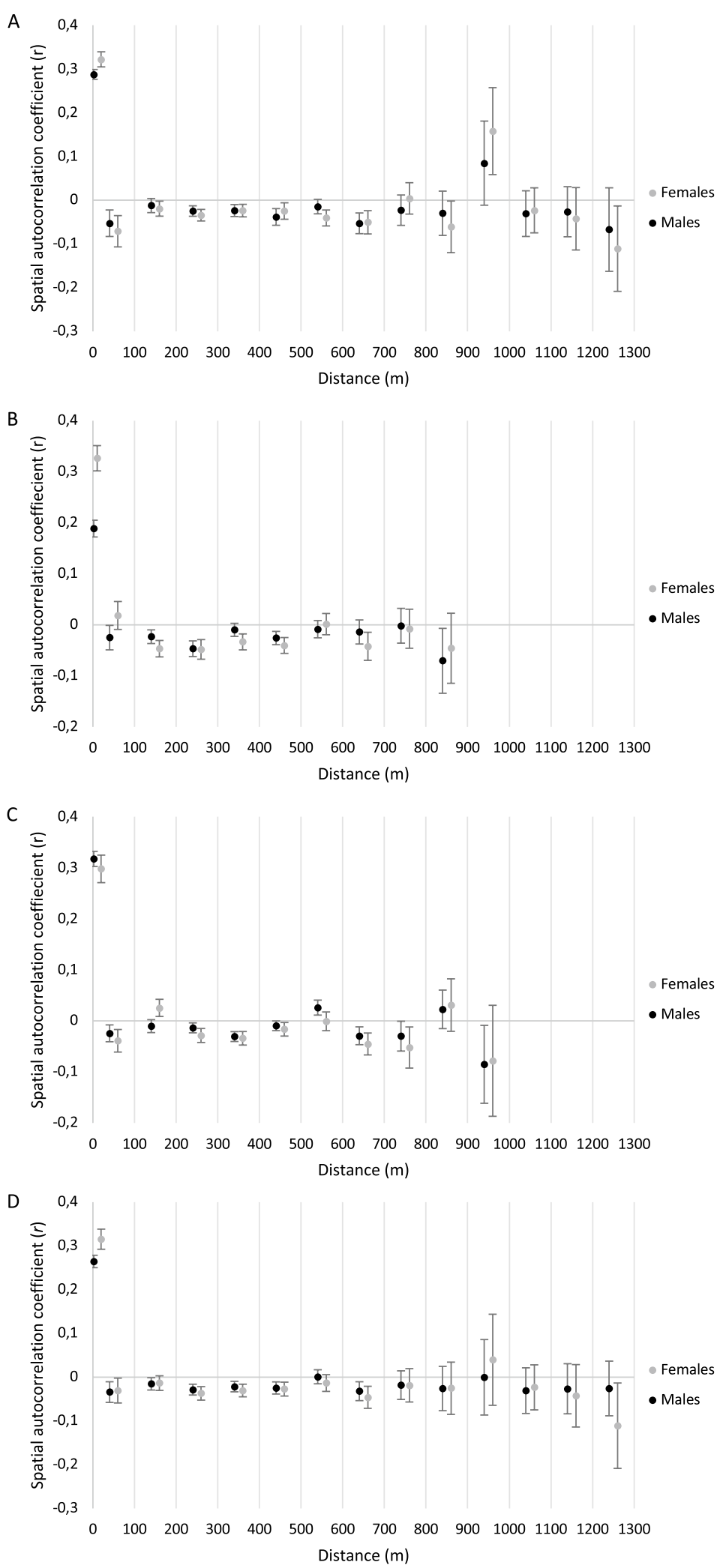

Fig. 4 (See legend on previous page.) 


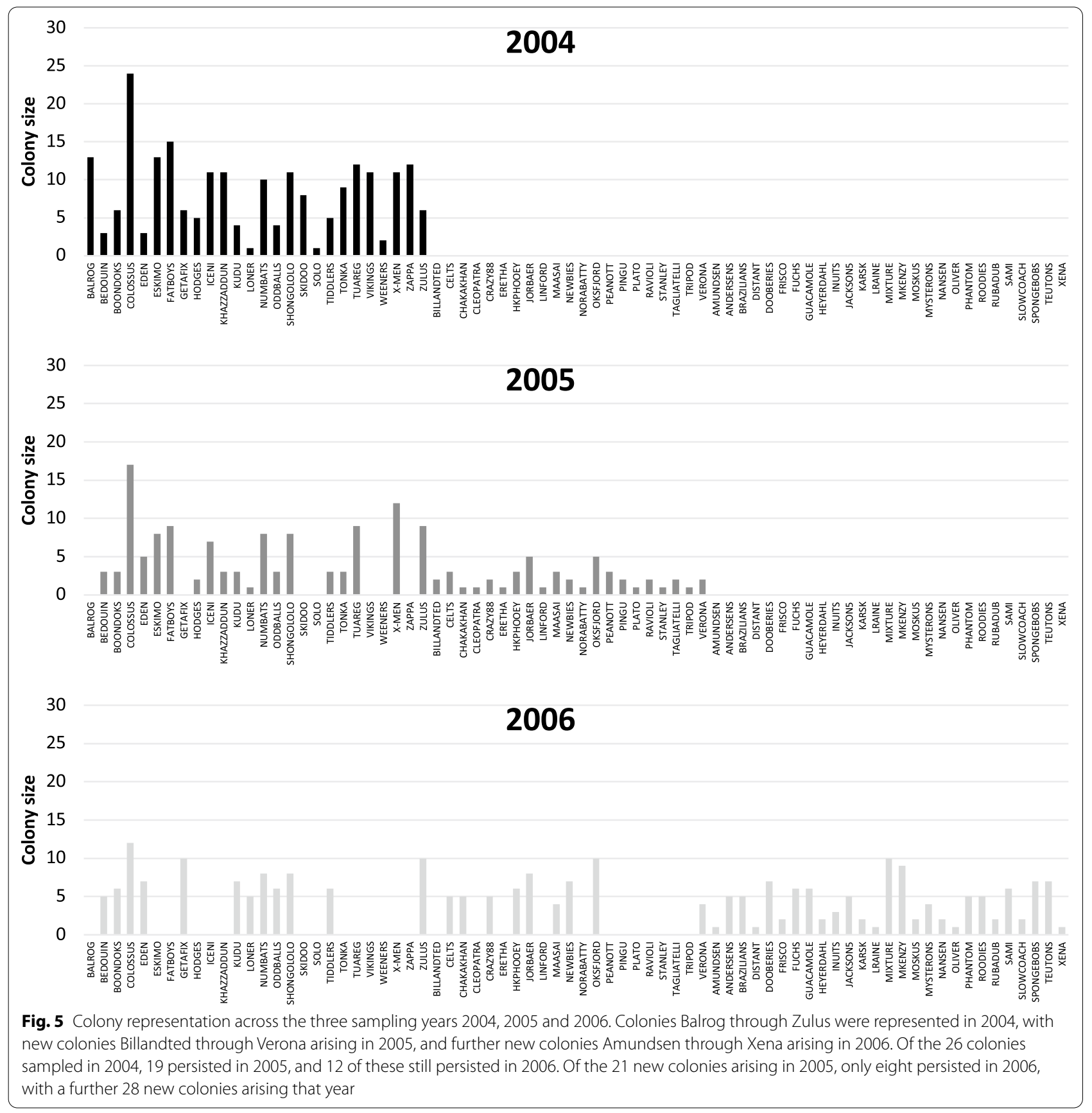

et al. [37] found that 121 (52 female, 69 male) out of 153 Fukomys damarensis dispersers (79\%) dispersed to establish new colonies, and that 28 (7 female, 21 male; 18\%) dispersed in coalition (along with other individuals of the same sex).

\section{Male-biased dispersal}

Both our observational data and genetic data are in line with male-biased dispersal in this species. We did not detect a skew in the number of male vs female dispersers, but present multiple lines of evidence that males typically disperse farther than females and are more likely than females to disperse to other established colonies, as opposed to founding new colonies. The observational data revealed that males dispersed on average $215 \mathrm{~m} \pm 180 \mathrm{~m}$, while females typically dispersed only $139 \mathrm{~m} \pm 104 \mathrm{~m}$, although this difference is non-significant. The genetic data were not powerful enough to 


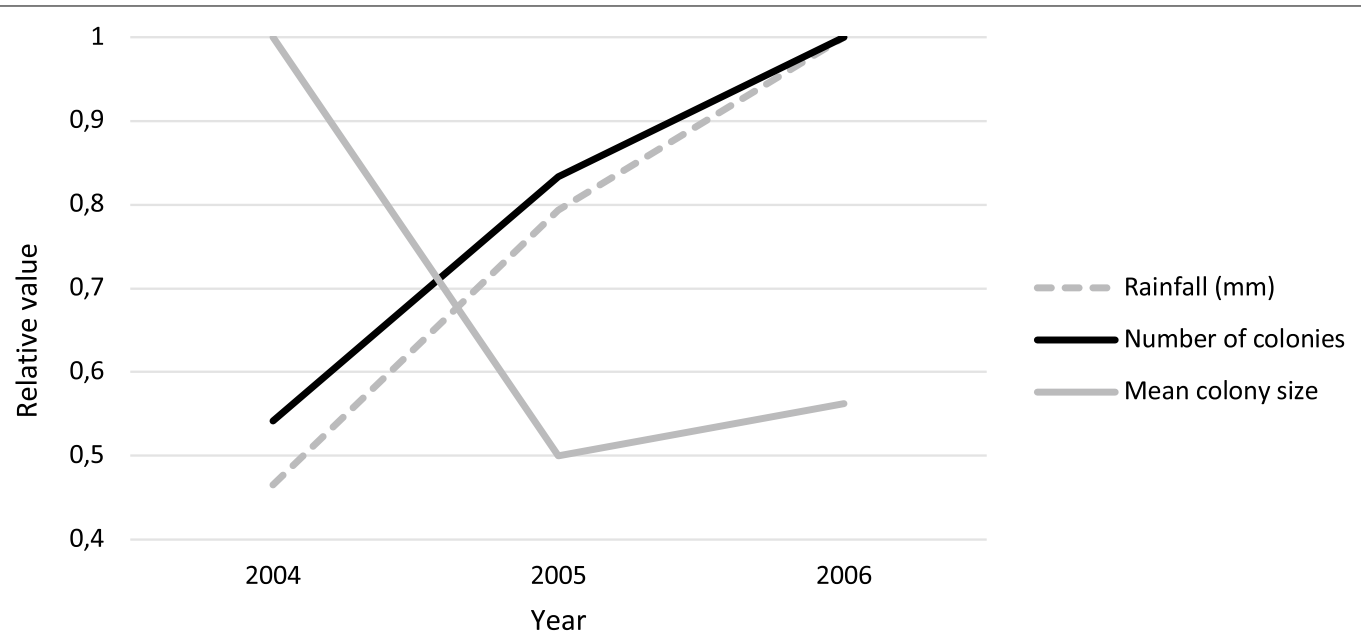

Fig. 6 Colony fission in response to rainfall. Y-axis values are shown as relative values, for each of three measurements (rainfall, number of colonies and mean colony size), i.e. as a proportion of the highest value in each given measurement category, e.g. in 2005 mean colony size was half (0.5) that of 2004. Colony numbers increase and sizes decrease with increasing annual rainfall between 2004 and 2006, indicative of colony fission along with subsequent colony growth through reproduction

accurately estimate $\sigma$ for females in any given year, however, we were able to estimate $\sigma$ for males in 2004, 2006 and across all 3 years. The mean predicted dispersal distance across all 3 years as inferred from the genetic data $(191 \mathrm{~m})$ is slightly lower than the mean estimate obtained from observational data for males $(215 \mathrm{~m} \pm 180 \mathrm{~m})$, but still substantially higher than the mean estimate for females $(139 \mathrm{~m} \pm 104 \mathrm{~m}$ ). Interestingly, of the 36 (19 male; 17 female) dispersal events, only four represented distances greater than $400 \mathrm{~m}$, and all of these were males. Female dispersals were typically associated with establishment of new colonies (e.g. colony fission events), with only $12 \%$ of dispersal events involving immigration into neighbouring colonies, whereas males were found to disperse more frequently into established colonies $(32 \%$ of male dispersal events). Another study has similarly demonstrated a high proportion of immigrants within established colonies of $F$. damarensis (4.3\% of nonbreeding colony members at Dordabis and $13.9 \%$ at Hotazel) and $F$. anselli (3.5\% at Lusaka), although this study did not specifically analyse the proportion of male vs. female immigrants.

Population-level $F_{\mathrm{ST}}$ analysis revealed a higher mean $F_{\mathrm{ST}}$ among females than males, indicating significantly greater genetic differentiation among females than males $(p<0.001)$, a pattern consistent with females being the more philopatric sex, and males being more dispersive. In 2004 and 2006, the difference between male and female $F_{\mathrm{ST}}$ became more pronounced with increasing spatial distance, consistent with the idea that males disperse over greater distances than females. Furthermore, spatial autocorrelation analysis indicated that on average, across all 3 years, intra-colony relatedness was significantly higher among females than males, while inter-colony relatedness was significantly lower. This is consistent with diminishing spatial genetic structure among males caused by long-distance male dispersal [44]. IBD was consistently more pronounced among females than males, consistent with the hypothesis that males are the more dispersive sex, and may disperse over greater distances than females.

The asymmetry of dispersal between the sexes observed in this study is somewhat consistent with the results of previous studies in social mole-rats. Genetic studies of the colony compositions of social mole-rats have shown that unrelated male non-breeders may be present in the colonies, suggesting that males may occasionally immigrate into colonies in the wild [65-67]. A study of Damaraland mole-rats that drowned in a channel suggested that males may more readily disperse than females [36]. Furthermore, a recent study of this species demonstrated that males disperse earlier (males: 371 days \pm 257 ; females: 411 days \pm 279 ) and more frequently (males: 96 individuals; females: 60 individuals) than females [37]. However, a recent study of Damaraland mole-rats from the Kalahari (including a population at the Kalahari Research Centre and the current population at Tswalu), found a higher number of females (31 out of 41 female dispersers, i.e. $76 \%$ ) taking on long-distance dispersal $(>250 \mathrm{~m}$ ) than males (27 out of 47 male dispersers, i.e. $57 \%$ ), although the number of male dispersers (47 individuals) was still higher overall than females (41 individuals) [57]. 
Several other studies of social mole-rat species have demonstrated that males may be more likely to disperse and may disperse further, whereas females may be more likely to undertake local dispersal to establish a territory in close proximity to their natal group [67-71].

\section{Effects of ecological constraints on dispersal and colony fission}

The compacted-sand substrate characterising the arid ecoregions inhabited by Damaraland mole-rats is extremely costly to work [72] and poses an ecological constraint that is thought to have favoured the evolution of delayed dispersal and cooperative breeding [25, 27, 29]. However, during periods of sustained rainfall, this constraint relaxes significantly, and the softened sands stimulate increased dispersal by subordinates of both sexes (either to immigrate to other existing colonies or to establish new colonies; [25, 36, 65]). Physiological suppression among subordinate females eases during the annual rains, when ecological constraints on dispersal are relaxed, despite the continued presence of the dominant female and in groups that contain no new immigrant males [40]. This is not only because the subordinate female's own chances of successful dispersal are higher, but because her chances of encountering mating opportunities with unrelated males are also increased $[25,36$, 65].

Our results demonstrate elevated dispersal and colony fission associated with increased rainfall. Although the mark-recapture data revealed only 36 "dispersal events", of which eight represented dispersals into established colonies, and 28 represented colony fissions, we observed a total of 49 newly established colonies across the 3-year sampling period, indicating that colony fission was greater than the mark-recapture data revealed. This is because "dispersal events" were only recorded if individuals were captured multiple times in different colonies, and some individuals may have evaded recapture, or been involved in colony fissions without having been previously captured in their natal colony. Colony fission in this population would involve individuals moving out of larger established colonies, in which they were most likely reproductively suppressed and involved in altruistic co-operative activities, to establish new colonies by means of colony genesis (pairing with an unrelated mate and establishing a new family group; [26]), thereby increasing the number of reproductively active animals in the overall population and consequently reducing reproductive skew. This finding supports the hypothesis [27] that environmental factors, such as rainfall, may play a significant role in the maintenance and/or disruption of reproductive skew in this species.
The structure of social groups in species with dispersal asymmetry can be described by two important parameters, i.e. the mean number and the mean size of the groups [1], although when considering hypotheses for the evolution of sociality, it is important to note that these life-history parameters depend directly upon environmental conditions. Resource availability (food, water, shelters, space), as well as abiotic conditions (climate) and biotic environment (density of predators, allospecific competition), directly influence birth and survival rates $[1,73]$. In social species with dispersal asymmetry and female philopatry, colony fission (e.g. involving a single male and female "budding" from their native colony into a neighbouring territory as described in our study, as opposed to long-distance dispersal occasionally undertaken predominantly by males), may be seen as a way for females to disperse. Through colony fission, females can leave the other females in their natal colony, their own relatives, and even their natal home range [1]. Moreover, the critical size for fission to occur is seldom reached under unfavourable demographic and environmental conditions (often characterising arid habitats), thus further driving delayed dispersal, colony growth and cooperative breeding and/or reproductive skew in these species.

\section{Methods}

\section{Study site and sampling}

Fukomys damarensis were live trapped at Tswalu Nature Reserve in the southern Kalahari $\left(27^{\circ} 12.855^{\prime} \mathrm{S}\right.$ $22^{\circ} 27.364^{\prime}$ E), South Africa using Hickman tunnel traps [74]. The $1700 \mathrm{~m} \times 500 \mathrm{~m}$ study site (Fig. 1) comprised a total of 74 temporally variable burrow systems, or colonies, which were sampled biannually (both in the wet and dry season) over a period of 3 consecutive years (2004-2006).

Active burrows were located by excavating one or two of the surface mounds that essentially radiate out from the centre of a colony like the spokes of a wheel. Excavated holes were left open with wire mesh inserted to prevent the entry of mole snakes and if further activity was observed in the form of animals blocking the excavated holes from the inside, traps were set after removal of the soil and mesh. Each colony was usually trapped from a single active hole, however, in some cases multiple holes in close proximity were found to belong to a single colony, which was determined by the presence of a single breeding pair. Breeding females were easily recognised by a perforate vagina and swollen teats, and breeding males by a stained mouth and bulging inguinal testes $(24$, as validated by 65 ), and the sexes were differentiated by the shape of their genitalia [24]. 
By continually running trap sites for multiple days and keeping trapped individuals in captivity (in plastic bins lined with fresh sand and provisioned with fresh sweet potato), entire colonies were trapped out. Colonies were considered "trapped out" when no further activity was noted for 2 consecutive days. Animals were marked for recapture in subsequent sampling periods using a unique toe-clipping system for identification. At the time of the study microchips and readers were not available for marking animals. Sex, mass, colony membership and GPS co-ordinates were recorded for each capture, and skin biopsies were stored for genetic analysis. After sampling, all individuals were returned to their burrow systems.

A total of 789 captures were made over the 3-year period, representing 486 unique individuals (excluding re-captures). Trapping sites differed between sampling periods, but individual identification of breeding females through mark-recapture facilitated identification of colonies, even if the burrow system had spatially shifted slightly between sampling periods, or if the sampling site represented a different portion of the burrow system. Hence GPS co-ordinates, representing the actual trap site, of some colonies differed slightly from 1 year to the next.

\section{Genotyping}

Whole genomic DNA was extracted from the collected tissue samples using the phenol/chloroform extraction method [75]. PCR amplification was carried out in 96-well plates (in total volumes of $10 \mu \mathrm{L}$ ) as follows: $0.5 \mathrm{U}$ of Super-Therm ${ }^{\circledR}$ DNA polymerase (Southern Cross Biotechnology), $1 \times$ Buffer (Southern Cross Biotechnology), $0.2 \mathrm{mM}$ of each of the four dNTP's (Promega) $1.5 \mathrm{pM}$ of each primer, approximately 20 ng of DNA and 0.6$1.5 \mathrm{mM} \mathrm{MgCl}{ }_{2}$ per reaction. Genotypes were generated on an ABI automated sequencer (Applied Biosystems) and analysed using GENEMAPPER Version 3.0 (Applied Biosystems).

Genotypic profiles were generated for all 486 captured individuals using seven genus-specific microsatellite loci (Table 1) after testing 11 loci developed by Burland et al. [76]. Of the 11 markers tested, two were omitted from subsequent analyses due to high levels of null alleles ( $\mathrm{CH} 1$ and $\mathrm{CH} 4)$, one due to poor amplification success in our study $(\mathrm{CH} 2)$, and one due to potential sex-linkage (DMR1). Modifications were made to three of the primer pairs (DMR1, DMR3 and DMR7) to facilitate co-loading on the ABI 3100 sequencer during GENESCAN ${ }^{\circledR}$ analyses. The loci were renamed to DMRN1, DMRN3 and DMRN7, respectively. Primers were $5^{\prime}$ fluorescent labelled (The Scientific Group). Allele frequencies, observed and expected heterozygosity were calculated for each marker using CERvus (Version 3.0.7), and assessed for Hardy-Weinberg equilibrium and the presence of null alleles.

\section{Dispersal estimates from mark-recapture data}

Mark-recapture data were collected throughout the study period (2004-2006), and dispersal events were recorded when the same individual was captured in more than one colony, either in the same sampling year, or in successive years. In some cases, dispersals within a sampling period were verified by recapture of that individual in the new colony in a subsequent sampling period, but this was not true for all dispersals, therefore we do not rule out the possibility that some of these recorded "dispersals" do not represent true dispersal events, but rather other movements or forays (such as unsuccessful breeding attempts, or simply foraging forays) [77], between colonies. However, since this study was conducted alongside a genetic investigation into gene flow, essentially reflecting only reproductively successful dispersal events, we considered

Table 1 Marker data for 7 microsatellite markers (Burland et al. [76]) employed in this study

\begin{tabular}{|c|c|c|c|c|c|c|c|c|c|}
\hline Marker & Dye & Size range & $\mathrm{T}_{\mathrm{A}}$ & $\begin{array}{l}\% \text { successfully } \\
\text { scored }\end{array}$ & $k$ & $H_{0}$ & $H_{\mathrm{E}}$ & HW & $F$ (null) \\
\hline $\mathrm{CH} 3$ & PET & $120-136$ & 54.6 & 82.7 & 13 & 0.864 & 0.872 & NS & 0.0046 \\
\hline DMR2 & 6-FAM & $151-169$ & 61.7 & 80.8 & 13 & 0.870 & 0.854 & NS & -0.0092 \\
\hline DMRN3 $^{\mathrm{a}}$ & VIC & $126-154$ & 57.6 & 90.3 & 13 & 0.841 & 0.869 & $* * *$ & 0.0183 \\
\hline DMR4 & NED & $205-225$ & $57-55$ & 83.2 & 19 & 0.796 & 0.825 & $* * *$ & 0.0210 \\
\hline DMR5 & 6-FAM & $244-274$ & $57-55$ & 78.3 & 18 & 0.809 & 0.777 & $*$ & -0.0222 \\
\hline DMR6 & NED & $123-147$ & $57-55$ & 87.2 & 10 & 0.465 & 0.866 & $* * *$ & 0.3040 \\
\hline DMRN7 $^{\mathrm{a}}$ & $\mathrm{VIC}$ & $132-148$ & 60.3 & 83.2 & 9 & 0.826 & 0.834 & $*$ & 0.0058 \\
\hline
\end{tabular}

$k$ : number of alleles in 486 genotyped individuals; $H_{\mathrm{O}}$ : observed heterozygosity; $H_{\mathrm{E}}$ : expected heterozygosity; $\mathrm{HW}$ : Hardy-Weinberg equilibrium; NS: not significant; $F$ (null): frequency of null alleles

${ }^{\text {a }}$ Newly designed $\mathrm{F}$ primers 


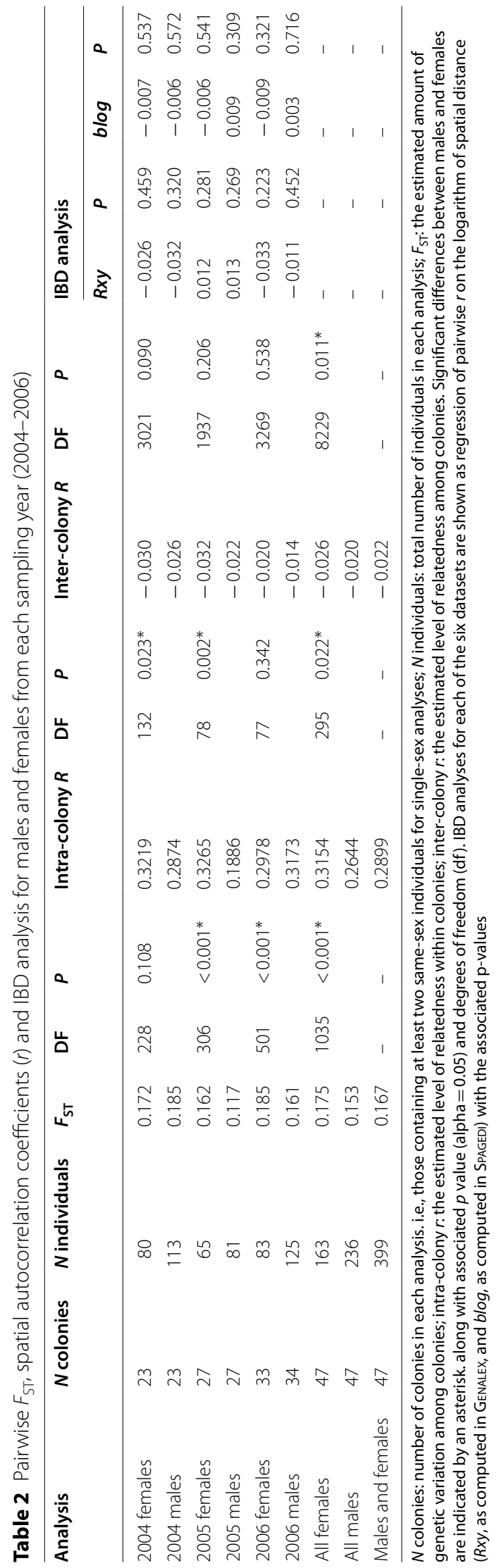


all recorded movements of individuals between colonies as relevant observational data. We also acknowledge that some dispersing individuals may have evaded recapture, and that our data thus represent only a subset of the total pool of dispersers within the population.

Body mass was used as an indicator of age; individuals weighing more than $80 \mathrm{~g}$ were deemed "adults", between 50 and $80 \mathrm{~g}$ "sub-adults", and less than $50 \mathrm{~g}$ "juveniles". These categories were used to distinguish natal dispersal from other dispersal events. GPS co-ordinates of capture sites were used to compute Euclidean dispersal distances. We assessed the significance of sex differences in mean dispersal distance, and frequency of dispersal across twelve distance classes of $50 \mathrm{~m}$ increments $(0-600 \mathrm{~m})$ using paired two-tailed t-tests, with the significance level set to 0.05 .

\section{Spatial genetic analysis}

Mark-recapture data was verified using genotypic data from seven microsatellite loci (Table 1). All 486 captured individuals were genotyped, but only the 399 individuals that passed the maximum missingness threshold of $50 \%$ were analysed. Thus, the 3 sampling years were analysed separately, with 193 individuals representing 23 colonies for 2004, 146 individuals representing 27 colonies for 2005 and 208 individuals representing 34 colonies for 2006 (Table 2). GPS co-ordinates were used to compute distances between colonies and between individuals, and 13 distance classes of $100 \mathrm{~m}$ increments were specified, with the first class representing distances up to $100 \mathrm{~m}$, and the final class representing distances between 1200 and $1300 \mathrm{~m}$ (the maximum distance between any two colonies in our study site was $1227 \mathrm{~m}$ ). Pairwise $F_{\text {ST }}$ [78] was estimated using a nested ANOVA [79] to assess spatial autocorrelation of genetic variance among colonies. In the case of cooperative breeders, $F_{\mathrm{ST}}$ represents the proportion of genetic variance that is partitioned among different social groups, or colonies. Low $F_{\mathrm{ST}}$ values imply that colonies are genetically similar, whereas high values suggest that colonies are genetically more distinct, and the lower the rate of gene flow between colonies, the higher the $F_{\mathrm{ST}}$ value. Since $F_{\mathrm{ST}}$ values depend not only on the amount of differentiation among populations, but also on the diversity within, which may be influenced by the choice of marker, there is no "rule" as to what constitutes high or low $F_{\mathrm{ST}}$. However, in broad terms $F_{\mathrm{ST}}<0.05$ may be considered as generally low genetic differentiation, $0.05-0.15$ as moderate, $0.15-0.25$ as great and $>0.25$ as very great, thus $F_{\mathrm{ST}}>0.15$ may generally be considered as significant differentiation [80, 81]. Sex-biased dispersal can be assessed by calculating $F_{\mathrm{ST}}$ separately for males and females, and the more philopatric sex is expected to show higher $F_{\mathrm{ST}}$ values [44]. Significance of differences in $F_{\mathrm{ST}}$ between colonies represented by males and females across all distance classes was assessed by permutation analysis as implemented in SPAGEDI v.1.5 (Spatial Pattern Analysis of Genetic Diversity; [82]), and paired t-tests.

In addition to population-level $F_{\mathrm{ST}}$, pairwise relatedness coefficients $(r ;[83])$ were used to analyse fine-scale spatial genetic structure by assessing spatial autocorrelation of relatedness among individuals. Significance of differences in relatedness among males vs. females across all distance classes (including within colonies) was once again tested by permutation analysis as implemented in SPAGEDi [82], and paired t-tests.

\section{Isolation by distance}

We used both SPAGeDi v.1.5 [82] and GenAlEx v.6.5 [84] to test isolation by distance (IBD) separately in males and females for each of the 3 sampling years. Pairwise relatedness coefficients $(r)$ and spatial distance matrices, as computed in SPAGEDI, were used to perform Mantel tests in Genalex. Significance was assessed using 999 random permutations in GENALEX, and 1000 permutations in SPAGEDI.

Theoretical models of IBD show that, under certain conditions, relatedness coefficients between individuals are expected to vary approximately linearly with the logarithm of the distance in a two-dimensional space, and with the linear distance in a one-dimensional space $[85,86]$. Thus, a negative regression, i.e. slope of the relationship between spatial distance and genetic relatedness reflects increasing IBD, and is expected to be steeper in the more philopatric sex, for which relatedness decreases more rapidly with increasing spatial distance.

\section{Dispersal estimates from genetic data}

The gene dispersal distance parameter, sigma $(\sigma)$, was estimated from the regression of pairwise relationship coefficients (or Rousset distance) on the logarithmic distance, with the assumption that genotypes come from a two-dimensional population at drift-dispersal equilibrium such that theoretical expectations of isolationby-distance models hold [82, 85, 86]. SPAGEDi uses an iterative procedure to determine $\sigma$ (the square root of half the mean square parent-offspring distance) and $\mathrm{Nb}$ (neighbourhood size) by regressing pairwise relationship coefficients on $\ln$ (distance) over a restricted distance range. The procedure requires an estimate of the effective population density, $D$, as well as $X$, the width of the distance range $\sigma$ to $X \sigma$ over which the regression is applied. Starting from a global regression slope 
$(b \log )$, the procedure consists in estimating $N b$ as $N b=-\left(1-F_{(1)}\right) / b \log$, where $F_{(1)}$ is the relationship coefficient between individuals for the first distance class (assumed to correspond to pairs of neighbours; the intracolony class is excluded), and $\sigma$ is estimated as $\sigma=[\mathrm{Nb} /$ $(2 \pi \cdot k \cdot D)]^{1 / 2}$. Then, restricting the regression $(b \log )$ to distances between $\sigma$ and $\mathrm{X} \sigma, N b$ and $\sigma$ are estimated again. This step is repeated until $\sigma$ converges, with up to 100 iterations [82]. Convergence is not ensured, in which case no estimate is provided.

\section{Rainfall data collection}

Rainfall data for Tswalu Kalahari Reserve were provided by the Tswalu Foundation. Dispersal and spatial genetic data were evaluated in light of the fluctuation in annual precipitation averages. Chi-squared tests were used to test the statistical significance of differences in frequency of dispersal and colony fission events between sampling periods (2004-2005 and 2005-2006), and thus the association of these parameters with annual rainfall.

\section{Supplementary Information}

The online version contains supplementary material available at https://doi. org/10.1186/s12862-021-01950-2.

Additional file 1: Table S1. Evidence of dispersal from mark-recapture data, indicating the colonies/capture sites for individuals that were captured at different sites in successive years, and the spatial distance associated with each dispersal event.

\section{Acknowledgements}

We thank Andrew Young for his contributions towards field trips and the acquisition of samples, and for commenting on the draft manuscript. We acknowledge Dylan Smith of the Tswalu Foundation for providing Tswalu rainfall data for the study period and the Oppenheimer family for permission to work on the Tswalu Nature Reserve. We thank Carel Oosthuizen for assistance with genotype scoring. We thank the reviewer for insightful comments on the draft manuscript.

\section{Authors' contributions}

Collected the samples: NCB LHB. Conceived and designed the experiments: SM PB LHB NCB. Generated the genotypic data: LHB. Analyzed the data: SM PB LHB. Contributed reagents/materials/analysis tools: NCB PB. Wrote the paper: SM. Commented on the draft manuscript: NCB PB. All authors read and approved the final manuscript.

\section{Funding}

This work was funded in part by the National Research Foundation (NRF) South African Research Chair Initiative (SARChl) Chair of Behavioural Ecology and Physiology (NCB), and by NRF grant (unique Grant Number 46995; PB). We also acknowledge the University of Pretoria and the aforementioned SARChl Chair for postdoctoral funding (SM). The opinions, findings and conclusions expressed in this publication are those of the authors and the NRF accepts no responsibility in this regard.

\section{Availability of data and materials}

The dataset supporting the conclusions of this article is available in Dryad [Microsatellite genotypes for Damaraland mole-rats (Fukomys damarensis) from the southern Kalahari] at https://doi.org/10.5061/dryad.8sf7m0ckq.

\section{Declarations}

Ethics approval and consent to participate

All animals were live-trapped and released after 2 days in captivity (in plastic bins lined with fresh sand and provisioned with fresh sweet potato). No animals were killed. All protocols were approved by the University of Pretoria ethics committee (EC050727-016) and complied with regulations stipulated in the Guidelines for the use of Animals in Research. This study is reported in accordance with ARRIVE guidelines.

\section{Consent for publication}

Not applicable.

\section{Competing interests}

The authors declare that they have no competing interests.

\section{Author details}

${ }^{1}$ Molecular Ecology and Evolution Programme, Department of Biochemistry, Genetics and Microbiology, University of Pretoria, Pretoria, South Africa. ${ }^{2}$ Department of Zoology and Entomology, University of Pretoria, Pretoria, South Africa. ${ }^{3}$ Mammal Research Institute (MRI), University of Pretoria, Pretoria, South Africa.

Received: 27 June 2021 Accepted: 26 November 2021

Published online: 08 December 2021

References

1. Lefebvre D, Ménard N, Pierre J-S. Modelling the influence of demographic parameters on group structure in social species with dispersal asymmetry and group fission. Behav Ecol Sociobiol. 2003;53(6):402-10.

2. Greenwood PJ. Mating systems, philopatry and dispersal in birds and mammals. Anim Behav. 1980;28(4):1140-62.

3. Clarke AL, Sæther B-E, Røskaft E. Sex biases in avian dispersal: a reappraisal. Oikos. 1997;79:429-38.

4. Chesser RK. Gene diversity and female philopatry. Genetics 1991;127(2):437-47.

5. Storz JF. Genetic consequences of mammalian social structure. J Mammal. 1999;80(2):553-69.

6. Pope TR. The influence of dispersal patterns and mating systems on genetic differentiation within and between populations of the red howler monkey (Alouatta seniculus). Evolution. 1992:46(4):1112-28.

7. van Noordwijk MA, Arora N, Willems EP, Dunkel LP, Amda RN, Mardianah $\mathrm{N}$, et al. Female philopatry and its social benefits among Bornean orangutans. Behav Ecol Sociobiol. 2012;66(6):823-34.

8. Castella V, Ruedi M, Excoffier L. Contrasted patterns of mitochondrial and nuclear structure among nursery colonies of the bat Myotis myotis. J Evol Biol. 2001;14(5):708-20.

9. Petit E, Mayer F. Male dispersal in the noctule bat (Nyctalus noctula): where are the limits? Proc R Soc Lond B. 1999;266(1430):1717-22.

10. Dobson FS, Smith AT, Gao WX. The mating system and gene dynamics of plateau pikas. Behav Proc. 2000;51(1-3):101-10.

11. Matocq MD, Lacey EA. Philopatry, kin clusters, and genetic relatedness in a population of woodrats (Neotoma macrotis). Behav Ecol. 2004;15(4):647-53.

12. McEachern M, Eadie JM, Van Vuren DH. Local genetic structure and relatedness in a solitary mammal, Neotoma fuscipes. Behav Ecol Sociobiol. 2007:61(9):1459-69.

13. Alexander RD. The evolution of social behavior. Annu Rev Ecol Syst. 1974:5(1):325-83.

14. Avilés L. Cooperation and non-linear dynamics: an ecological perspective on the evolution of sociality. Evol Ecol Res. 1999;1(4):459-77.

15. Pulliam HR. Living in groups: is there an optimal group size? Behav Ecol Evol Approach. 1984:122-47.

16. Avilés L. Nomadic behaviour and colony fission in a cooperative spider: life history evolution at the level of the colony? Biol J Lin Soc. 2000;70(2):325-39. 
17. Franks NR, Fletcher CR. Spatial patterns in army ant foraging and migration: Eciton burchelli on Barro Colorado Island, Panama. Behav Ecol Sociobiol. 1983;12(4):261-70.

18. Brett RA. The population structure of naked mole-rat colonies. In: The biology of the naked mole-rat. Princeton: Princeton University Press; 1991. p. 97-136

19. Sherman PW, Jarvis JU, Alexander RD. The biology of the naked mole-rat. Princeton: Princeton University Press; 2017.

20. Armitage KB. Social dynamics of mammals: reproductive success, kinship and individual fitness. Trends Ecol Evol. 1987;2(9):279-84.

21. Blumstein DT, Armitage KB. Life history consequences of social complexity a comparative study of ground-dwelling sciurids. Behav Ecol. 1998;9(1):8-19.

22. Chepko-Sade BD, Sade DS. Patterns of group splitting within matrilineal kinship groups: a study of social group structure in Macaca mulatta (Cercopithecidae: Primates). Behav Ecol Sociobiol. 1979;5:67-86.

23. Dittus WP. Group fission among wild toque macaques as a consequence of female resource competition and environmental stress. Anim Behav. 1988;36(6):1626-45.

24. Bennett N, Faulkes C. African mole-rats: ecology and eusociality. Cambridge: Cambridge University Press; 2000. p. 273.

25. Jarvis J, Bennett N. Eusociality has evolved independently in two genera of bathyergid mole-rats-but occurs in no other subterranean mammal. Behav Ecol Sociobiol. 1993;33(4):253-60.

26. Bennett N, Jarvis J. The social structure and reproductive biology of colonies of the mole-rat, Cryptomys damarensis (Rodentia, Bathyergidae). J Mammal. 1988;69(2):293-302.

27. Jarvis J, O'Riain M, Bennett N, Sherman P. Mammalian eusociality: a family affair. Trends Ecol Evol. 1994;9(2):47-51.

28. Bennett N, Faulkes C, Molteno A. Reproductive suppression in subordinate, non-breeding female Damaraland mole-rats: two components to a lifetime of socially induced infertility. Proc Biol Sci. 1996;263(1376):1599-603.

29. Faulkes C, Bennett N, Bruford MW, O'brien H, Aguilar G, Jarvis J. Ecological constraints drive social evolution in the African mole-rats. Proc R Soc Lond Ser B Biol Sci. 1997;264(1388):1619-27.

30. Jarvis J. Ecological studies on Heterocephalus glaber, the naked mole-rat Kenya Nat Geogr Soc Res Rep. 1985;20:429-37.

31. Lovegrove B. The evolution of eusociality in molerats (Bathyergidae): a question of risks, numbers, and costs. Behav Ecol Sociobiol. 1991;28(1):37-45.

32. Lovegrove B, Wissel C. Sociality in molerats: metabolic scaling and the role of risk sensitivity. Oecologia. 1988;74(4):600-6.

33. Lövy M, Šklíba J, Burda H, Chitaukali W, Šumbera R. Ecological characteristics in habitats of two African mole-rat species with different social systems in an area of sympatry: implications for the mole-rat social evolution. J Zool. 2012;286(2):145-53.

34. Cooney R, Bennett NC. Inbreeding avoidance and reproductive skew in a cooperative mammal. Proc R Soc Lond B. 2000;267(1445):801-6.

35. Molteno AJ, Bennett NC. Rainfall, dispersal and reproductive inhibition in eusocial Damaraland mole-rats (Cryptomys damarensis). J Zool. 2002;256(04):445-8.

36. Hazell RWA, Bennett NC, Jarvis JUM, Griffin M. Adult dispersal in the cooperatively breeding Damaraland mole-rat, Cryptomys damarensis: a case study from the Waterberg region of Namibia. J Zool. 2000;252(1):19-26.

37. Torrents-Ticó M, Bennett NC, Jarvis JU, Zöttl M. Sex differences in timing and context of dispersal in Damaraland mole-rats (Fukomys damarensis). J Zool. 2018:306(4):252-7.

38. Burland T, Bennett N, Jarvis J, Faulkes C. Eusociality in African molerats: new insights from patterns of genetic relatedness in the Damaraland mole-rat (Cryptomys damarensis). Proc R Soc Lond B. 2002:269(1495):1025-30.

39. Spinks AC, Jarvis JUM, Bennett NC. Comparative patterns of philopatry and dispersal in two common mole-rat populations: implications for the evolution of mole-rat sociality. J Anim Ecol. 2000;69(2):224-34.

40. Young AJ, Oosthuizen MK, Lutermann H, Bennett NC. Physiological suppression eases in Damaraland mole-rat societies when ecological constraints on dispersal are relaxed. Horm Behav. 2010;57(2):177-83.

41. Francioli Y, Thorley J, Finn K, Clutton-Brock T, Zöttl M. Breeders are less active foragers than non-breeders in wild Damaraland mole-rats. Biol Let. 2020;16(10):20200475
42. Faulkes CG, Bennett NC. Damaraland and naked mole-rats: convergence of social evolution. Cooperative Breed Vertebr. 2016;1:338-52.

43. Banks SC, Peakall ROD. Genetic spatial autocorrelation can readily detect sex-biased dispersal. Mol Ecol. 2012;21(9):2092-105.

44. Goudet J, Perrin N, Waser P. Tests for sex-biased dispersal using biparentally inherited genetic markers. Mol Ecol. 2002;11(6):1103-14.

45. Peakall R, Ruibal M, Lindenmayer DB. Spatial autocorrelation analysis offers new insights into gene flow in the Australian bush rat, Rattus fuscipes. Evolution. 2003;57(5):1182-95.

46. Ribeiro A, Lloyd P, Feldheim K, Bowie RC. Microgeographic socio-genetic structure of an African cooperative breeding passerine revealed: integrating behavioural and genetic data. Mol Ecol. 2012;21(3):662-72.

47. Rollins LA, Browning LE, Holleley CE, Savage JL, Russell AF, Griffith SC. Building genetic networks using relatedness information: a novel approach for the estimation of dispersal and characterization of group structure in social animals. Mol Ecol. 2012:21(7):1727-40.

48. Gardner A. Sex-biased dispersal of adults mediates the evolution of altruism among juveniles. J Theor Biol. 2010;262(2):339-45.

49. Johnstone RA, Cant MA. Sex differences in dispersal and the evolution of helping and harming. Am Nat. 2008;172(3):318-30.

50. Young AJ, Bennett NC. Intra-sexual selection in cooperative mammals and birds: why are females not bigger and better armed? Philos Trans $\mathrm{R}$ Soc B Biol Sci. 2013;368(1631):20130075.

51. Berg EC, Eadie J, Langen TA, Russell A. Reverse sex-biased philopatry in a cooperative bird: genetic consequences and a social cause. Mol Ecol. 2009;18(16):3486-99.

52. Clutton-Brock T, Russell A, Sharpe L, Young A, Balmforth Z, Mcllrath G Evolution and development of sex differences in cooperative behavior in meerkats. Science. 2002;297(5579):253-6.

53. Cockburn A, Osmond HL, Mulder RA, Double MC, Green DJ. Demography of male reproductive queues in cooperatively breeding superb fairywrens Malurus cyaneus. J Anim Ecol. 2008;77(2):297-304.

54. Creel SR, Rabenold KN. Inclusive fitness and reproductive strategies in dwarf mongooses. Behav Ecol. 1994;5(3):339-48.

55. Emlen ST. Evolution of cooperative breeding in birds and mammals, 2nd edn. In: Krebs JR, Davies NB, editors. Behavioural ecology. Oxford, UK: Blackwell Publications; 1991. p. 305-55.

56. Holekamp KE, Smith JE, Strelioff CC, Van Horn RC, Watts HE. Society, demography and genetic structure in the spotted hyena. Mol Ecol. 2012;21(3):613-32.

57. Finn KT. Potential use of a magnetic compass during long-distance dispersal in a subterranean rodent. J Mammal. 2021;102(1):250-7.

58. Young AJ, Monfort SL. Stress and the costs of extra-territorial movement in a social carnivore. Biol Lett. 2009;5(4):439-41.

59. Young AJ, Carlson AA, Clutton-Brock T. Trade-offs between extraterritorial prospecting and helping in a cooperative mammal. Anim Behav. 2005;70(4):829-37.

60. Lehmann L, Rousset F. How life history and demography promote or inhibit the evolution of helping behaviours. Philos Trans R Soc B Biol Sci. 2010;365(1553):2599-617.

61. Hatchwell BJ. Cryptic kin selection: kin structure in vertebrate populations and opportunities for kin-directed cooperation. Ethology. 2010;116(3):203-16.

62. Koenig WD, Haydock J. Incest and incest avoidance. In: Ecology and evolution of cooperative breeding in birds. New York: Cambridge University Press; 2004. p. 142-56.

63. Yáber MC, Rabenold KN. Effects of sociality on short-distance, femalebiased dispersal in tropical wrens. J Anim Ecol. 2002;71:1042-55.

64. Kokko H, Ekman J. Delayed dispersal as a route to breeding: territorial inheritance, safe havens, and ecological constraints. Am Nat. 2002;160(4):468-84

65. Burland T, Bennett NC, Jarvis JUM, Faulkes CG. Colony structure and parentage in wild colonies of co-operatively breeding Damaraland molerats suggest incest avoidance alone may not maintain reproductive skew. Mol Ecol. 2004;13(8):2371-9.

66. Bishop J, Jarvis J, Spinks A, Bennett N, O'ryan C. Molecular insight into patterns of colony composition and paternity in the common mole-rat Cryptomys hottentotus hottentotus. Mol Ecol. 2004;13(5):1217-29.

67. Patzenhauerová H, Šklíba J, Bryja J, Šumbera R. Parentage analysis of Ansell's mole-rat family groups indicates a high reproductive skew 
despite relatively relaxed ecological constraints on dispersal. Mol Ecol. 2013;22(19):4988-5000.

68. Šumbera R, Mazoch V, Patzenhauerová H, Lövy M, Šklíba J, Bryja J, et al. Burrow architecture, family composition and habitat characteristics of the largest social African mole-rat: the giant mole-rat constructs really giant burrow systems. Acta Theriol. 2012;57(2):121-30.

69. O'Riain MJ, Jarvis JU, Faulkes CG. A dispersive morph in the naked molerat. Nature. 1996;380(6575):619-21.

70. Lövy M, Šklíba J, Šumbera R. Spatial and temporal activity patterns of the free-living giant mole-rat (Fukomys mechowii), the largest social bathyergid. PLoS ONE. 2013;8(1): e55357.

71. Braude S. Dispersal and new colony formation in wild naked molerats: evidence against inbreeding as the system of mating. Behav Ecol. 2000;11(1):7-12.

72. Lovegrove B. The cost of burrowing by the social mole rats (Bathyergidae) Cryptomys damarensis and Heterocephalus glaber: the role of soil moisture. Physiol Zool. 1989;62(2):449-69.

73. Arnold KE, Owens IP. Cooperative breeding in birds: a comparative test of the life history hypothesis. Proc R Soc Lond B. 1998;265(1398):739-45.

74. Hickman GC. A live-trap and trapping technique for fossorial mammals. S Afr J Zool. 1979;14:9-12.

75. Sambrook J, Russell DW. Purification of nucleic acids by extraction with phenol:chloroform. Cold Spring Harb Protoc. 2006. https://doi.org/10. 1101/pdb.prot093450.

76. Burland T, Bishop JM, O'ryan C, Faulkes CG. Microsatellite primers for the African mole-rat genus Cryptomys and cross-species amplification within the family Bathyergidae. Molec Ecol Notes. 2001;1 (4):311-4.

77. Šklíba J, Mazoch V, Patzenhauerová H, Hrouzková E, Lövy M, Kott O, et al. A maze-lover's dream: burrow architecture, natural history and habitat characteristics of Ansell's mole-rat (Fukomys anselli). Mamm Biol. 2012;77(6):420-7.

78. Wright S. Evolution in Mendelian populations. Genetics. 1931;16(2):97-159.

79. Michalakis Y, Excoffier L. A generic estimation of population subdivision using distances between alleles with special reference for microsatellite loci. Genetics. 1996;142(3):1061-4.

80. Hartl DL, Clark AG. Principles of population genetics. Sunderland: Sinauer; 1997.

81. Frankham R, Ballou JD, Briscoe DA. Introduction to conservation genetics. 2nd ed. Cambridge: Cambridge University Press; 2010.

82. Hardy OJ, Vekemans X. SPAGeDi: a versatile computer program to analyse spatial genetic structure at the individual or population levels. Mol Ecol Notes. 2002;2(4):618-20.

83. Queller DC, Goodnight KF. Estimating relatedness using genetic markers. Evolution. 1989;43(2):258-75.

84. Peakall R, Smouse P. GenAlEx 6.5: genetic analysis in Excel. Population genetic software for teaching and research —an update. Bioinformatics. 2012. https://doi.org/10.1111/j.1471-8286.2005.01155.x.

85. Hardy OJ, Vekemans X. Isolation by distance in a continuous population: reconciliation between spatial autocorrelation analysis and population genetics models. Heredity. 1999;83(2):145-54.

86. Rousset F. Genetic differentiation and estimation of gene flow from F-statistics under isolation by distance. Genetics. 1997;145(4):1219-28.

\section{Publisher's Note}

Springer Nature remains neutral with regard to jurisdictional claims in published maps and institutional affiliations.
Ready to submit your research? Choose BMC and benefit from

- fast, convenient online submission

- thorough peer review by experienced researchers in your field

- rapid publication on acceptance

- support for research data, including large and complex data types

- gold Open Access which fosters wider collaboration and increased citations

- maximum visibility for your research: over $100 \mathrm{M}$ website views per year

At BMC, research is always in progress.

Learn more biomedcentral.com/submissions 